\title{
Telemedicine in respiratory medicine
}

\begin{abstract}
The development of techniques to acquire respiratory signals is still in its infancy. While various signals such as pulse Oximetry are able to be acquired in real time and even be transmitted electronically. The development of equipment which allows keep track of biological signals associated with breathing and Household ventilation equipment capable of monitoring compliance monitoring and treatment, sometimes fails to join a group of pilot projects under. It is the task of specialists in the respiratory area, biomedical engineers and patients themselves: to design, build and test systems and machines that bring the future of telemedicine respiratory medicine.
\end{abstract}

Volume 2 Issue 2 - 2015

\section{Jose Bruno De Lema}

High Complexity Foundation, Spain

Correspondence: Jose Bruno De Lema, High Complexity Foundation, Paseo de la montana \# I 4 bajo. granollers 08402 , Pereira, Barcelona, Spain, Email delemalarre@gmail.com

Received: February 24, 2015 | Published: February 28, 2015

Keywords: telemedicine, respiratory medicine, smartphone

Abbreviations: ADSL, asymmetric digital subscriber line; $3 \mathrm{G}$, third generation of mobile tele communications technology; 4G, fourth generation of mobile tele communications technology; CPAP, continuous positive airway pressure, ICT, information and communication technologies

\section{Introduction}

It is possible that the development of new technologies of information and communication technologies (ICT), is the new paradigm in the way of making a medical practice. Thinking about health care, focusing on the services of a certain degree of complexity, focus on the largest medical centers. The decentralization of services, it is so difficult to understand and Implement both physicians and patients themselves. Telemetry services can be a pathway for remote monitoring of patients, as a first phase. Later in the evaluation and monitoring by telemetry also means. ${ }^{1}$ In respiratory medicine, recording signals or as ventilation measures pulse Oximetry, is a reality, reviews having a group of instruments, while household telemetry applications. Still, the ranging possibilities of a set of pulse Oximetry equipment, ventilatory patterns, etc.; They are present as a performance option but go unnoticed by Implementing the test or treatment. $^{2}$

Currently sleep disorders are the leading telemedicine area; meet a number of requirements that make them very interesting.

i. Set of conditions of a high cost in diagnosis and treatment.

ii. Early intervention treatment systems Improves quality of life and secondary costs of a patient in more advanced stages of the disease.

iii. A high prevalence in the overall population. ${ }^{3}$

iv. The sleep disorders and Those of respiratory impairment from Cheyne-Stokes syndrome, apnea/ obstructive sleep apnea hypo or all Involving the use of ventilatory support: such as chronic obstructive pulmonary disease, chronic respiratory failure. Implement information and communication technologies (ICT), with a level of utility to the fore. Respiratory diseases during sleep where telemedicine is an area has great potential, Hence it the prevalence of these disorders, and the fact that they are basically chronic diseases. ${ }^{4,5}$

Possible applications of telemedicine are themselves respiratory sleep disorders (apnea/obstructive sleep apnea and Cheyne-Stokes hiccups). For nocturnal respiratory diseases or disturbances require ventilatory support during sleep: such as chronic respiratory failure due to neuromuscular disorders and/or chest, chronic obstructive pulmonary disease or hypoventilation syndrome/obesity. So far there have been experiences of telemetric transmission of physiological signals during sleep or home mechanical ventilation. There are attempts to use telemedicine to monitor compliance with treatment and make adjustments in real-time with continuous positive airway systems airway pressure (CPAP). ${ }^{6,7}$

It is always said the costs and complications of these applications are a problem of technical and economic nature, since it requires structures in computer engineering, since it is not only the collection of data by the device, but a data transmitter-through system mainly the $3 \mathrm{G}$ or $4 \mathrm{G}$ or simply ADSL systems using methods. There does not end the problem but more evident becomes when we consider that the centers where is received such information must have adequate reception and interpretation of information structures. Current efforts focus on equipment type smart phone.

Another aspect is the current user that is: sometimes far more reluctant to use telemetry systems, since the vast majority of target patients are not Users of computer systems, for being old or culture or even economic aspects. But all are not problems in respiratory telemedicine, in fact there are data acquisition systems CPAP apparatus, pulse Oximetry, etc. Be transmitted by a mobile phone. Even send information via mobile phone and modify forms of measures or parameters fans. It is more likely that your application and faster, than it has-been so far Being more modest in our claims-of telemedicine, more feasible. ${ }^{8-11}$

\section{Acknowledgements}

None.

\section{Conflict of interest}

The author declares no conflict of interest.

\section{References}

1. Commission of the European Communities. e-Health - making healthcare better for European Citizens: an action plan for a European e-Health Area.

2. Duplaga M, Soja J, Cala, Leszczuk M, et al. The impact of teleconsultations at a referential focus on the management of pulmonary patients. Stud Health Technol Inform. 2004;105:92-99. 
3. Whitten P, Mickus M. Home telecare for COPD/CHF patients: outcomes and perceptions. $J$ Telemed Telecare. 2007;13(2):69-73.

4. Vitacca M, Assoni G, Pizzocaro P, et al. A pilot study of nurse-led, home monitoring for patients With chronic respiratory failure and mechanical ventilation With assistance. J Telemed Telecare. 2006;12(7):337-342.

5. Vitacca M, Guerra A, Assoni G, et al. Followed Weaning from mechanical ventilation at home with the aid of a telemedicine program. Telemed $J$ E Health. 2007;13(4):445-449.

6. Kayyali HA, Weimer S, Frederick C, et al. Remotely Attended home monitoring of sleep disorders. Telemed J E Health. 2008;14(4):371-374.

7. Taylor, Eliasson A, Andrada T, et al. The role of telemedicine in CPAP compliance for patients With obstructive sleep apnea syndrome. Sleep Breath. 2006;10(3):132-138.
8. Ishida R, Yonezawa Y, Maki H, et al. A wearable, mobile phone-based respiration monitoring syste $\mathrm{m}$ for sleep apnea syndrome detection. Biomed SciInstrum. 2005;41:289-293.

9. Farré R, R Dellaca, Govoni L, et al. Home CPAP titration using Realtime telemetry. Bronconeumol. 2008;44(Suppl):204-5.

10. Koch S. Home telehealth - Current state and future trends. Int $J$ Med Inform. 2006;75(8):565-576.

11. Bria WF. Applied medical informatics for the chest physician: information you can use - Part 3. Chest. 2006;129(4):1057-1060. 